\title{
TITLE:
}

\section{Rotary motion driven by a direct current electric field}

\section{$\operatorname{AUTHOR}(\mathrm{S}):$}

Takinoue, Masahiro; Atsumi, Yu; Yoshikawa, Kenichi

\section{CITATION:}

Takinoue, Masahiro ... [et al]. Rotary motion driven by a direct current electric field. Applied Physics Letters 2010, 96(10): 104105.

ISSUE DATE:

2010-03-12

URL:

http://hdl.handle.net/2433/128847

RIGHT:

(C) 2010 American Institute of Physics 


\title{
Rotary motion driven by a direct current electric field
}

\author{
Masahiro Takinoue, ${ }^{a}$ Yu Atsumi, and Kenichi Yoshikawa ${ }^{\text {b) }}$ \\ Department of Physics, Graduate School of Science, Kyoto University, Kitashirakawa-Oiwake-cho, \\ Sakyo-ku, Kyoto 606-8502, Japan
}

(Received 7 December 2009; accepted 1 February 2010; published online 12 March 2010)

\begin{abstract}
We report the rotary motion of an aqueous microdroplet in an oil phase under a stationary direct current electric field. A droplet exhibits rotary motion under a suitable geometrical arrangement of positive and negative electrodes. Rotary motion appears above a certain critical electric potential and its frequency increases with an increase in the potential. A simple theoretical model is proposed to describe the occurrence of this rotary motion, together with an argument for the future expansion of this micro rotary motor system. (C) 2010 American Institute of Physics. [doi:10.1063/1.3358385]
\end{abstract}

Controlling the motion of micrometer-scale objects has been one of the attractive research subjects in applied physics. ${ }^{1}$ At a micrometer scale, the Reynolds number is very low; the inertia of an object does not play an important role and its motions are immediately damped by environmental viscosity. Thus, some driving energy has to be supplied to propel an object sustainably. Therefore, the motions of micro-objects have raised important issues in nonlinear and nonequilibrium systems. In addition, the ability to propel and control micro-objects, including biomacromolecules and cells, is important in applied physics and biophysics research, and in microelectromechanical system (MEMS) and micro-total analysis systems ( $\mu$ TAS) technology, and many suitable techniques have been actively developed, e.g., optical tweezers, ${ }^{2}$ electrophoresis and dielectrophoresis, ${ }^{3-5}$ microfluidic devices, ${ }^{4,6,7}$ bacteria-driven motors, ${ }^{8,9}$ motor proteins, ${ }^{10,11}$ etc.

In this letter, we report a method for generating rotary motion for a dielectric micro-object in an oil phase under a stationary direct current (dc) electric field. In particular, we focus on the rotary motion of a water-in-oil (w/o) microdroplet. To date, the occurrence of the linear periodic motion of a w/o microdroplet has been reported by some research groups. ${ }^{12-16}$ Most of them have reported one-dimensional back-and-forth motion under dc electric potential without any switching unit. However, there have been no reports on higher-dimensional cyclic motion under a constant electric field. At least two-dimensional cyclic motion is required to extract work and energy from microscale motion. ${ }^{1}$ The rotary motion of dielectric micro-objects that we report here may be useful for the development of a simple and useful strategy for controlling micro-objects.

A schematic illustration of the experimental setup is shown in Fig. 1. A water droplet was suspended in mineral oil on a glass slide, and constant voltage was applied to the droplet using cone-shaped tungsten electrodes [Fig. 1(a)]. These electrodes were placed at the same level [Fig. 1(a)] but noncoaxially [Fig. 1(b)]. Droplet motion was observed using an optical microscope (KEYENCE VH-Z75, Japan).

The w/o droplet was produced using a vortex mixer as follows. We prepared mineral oil including surfactant:

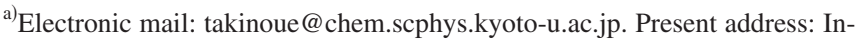
stitute of Industrial Science, The University of Tokyo, 4-6-1 Komaba, Meguro-ku, Tokyo 153-8505, Japan.

${ }^{b)}$ Electronic mail: yoshikaw @ scphys.kyoto-u.ac.jp.
}

$10 \mu \mathrm{M}$ surfactant, dioleoylphosphatidylcholine (DOPC) (Wako Pure Chemical Industries, Japan), was solved in mineral oil (Nacalai Tesque, Japan) by $90 \mathrm{~min}$ sonication at $50{ }^{\circ} \mathrm{C} .2 \mu \mathrm{l}$ ultrapure water (Millipore, Japan) was added to $200 \mu \mathrm{l}$ of the prepared mineral oil, and then agitated by a vortex mixer for approximately $3 \mathrm{~s}$.

The motions of a water droplet are shown in the left snapshot images of Figs. 2(a)-2(d). When the applied voltage was low $(V=20 \mathrm{~V})$ [Fig. 2(a)], the droplet wandered and escaped from the dielectric trapping force (Mode I). If the applied voltage exceeded a critical voltage, the droplet started to move cyclically [Figs. 2(b)-2(d)]. First, the droplet was attracted to the top of either electrode, and then was repelled from the electrode and moved toward the opposite electrode. This motion was sustained. The droplet motion followed a unidirectional circular trajectory that did not depend on the initial position of the droplet; i.e., the trajectory of the rotary motion was a kind of limit cycle. This rotary motion was seen not only with one water droplet but also with two or more droplets. The data of the rotary motion of two water droplets are shown in a supplementary material. ${ }^{17}$

In the experiments, we observed two following types of behavior for rotary motion: simple rotary motion (Mode II) and rotary motion with bouncing on the electrodes (Mode

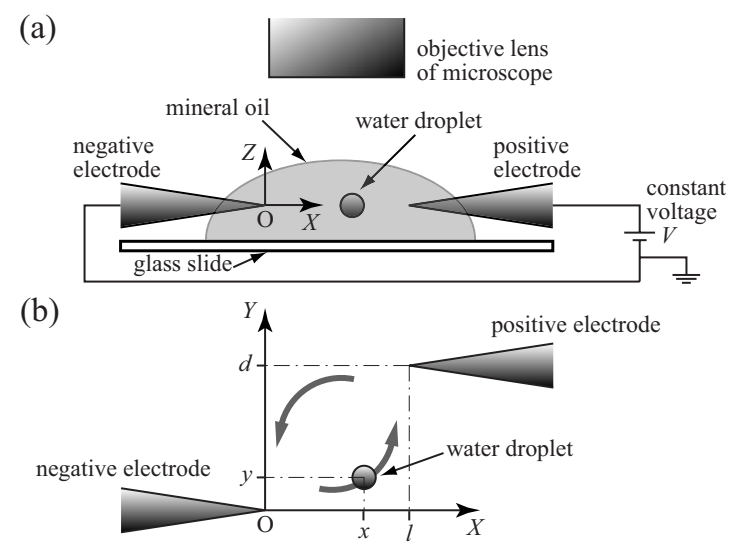

FIG. 1. (a) Side view of the experimental setup. Mineral oil including water droplets was placed on a glass slide and electrodes were inserted into the oil. The experiments were observed from above with a microscope. $V$ : Applied constant voltage between the electrodes. $X-Z$ coordinates: Vertical plane. $O$ (origin): Top of the negative electrode. (b) Overhead view (microscope view) of the experimental setup. $X-Y$ coordinates: Horizontal plane. $x$ $=(x, y)$ : Center of the water droplet. $(l, d)$ : Top of the positive electrode. 
(a)

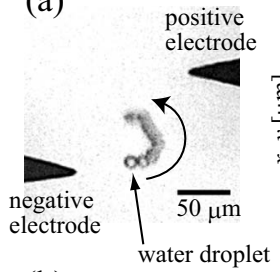

(b)

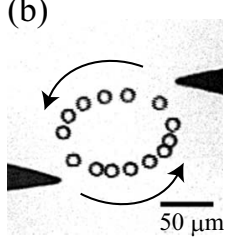

(c)

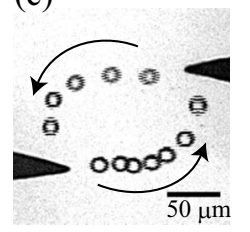

(d)
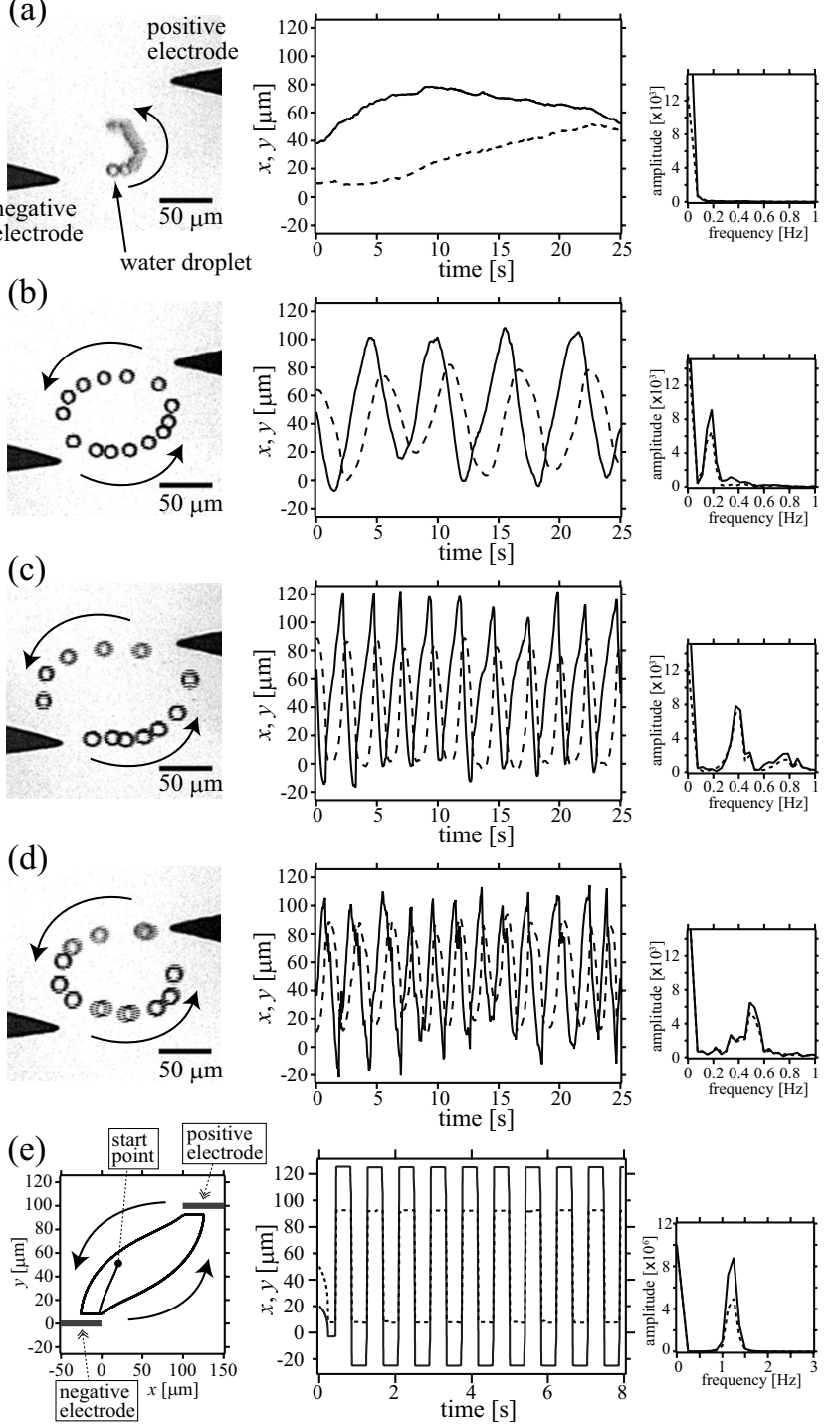

(a)

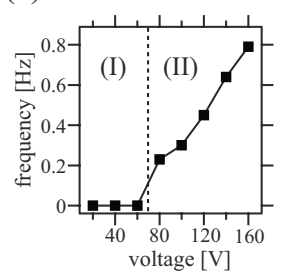

(d)

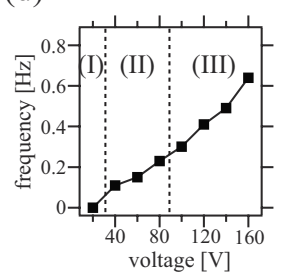

$(\mathrm{g})$ (b)

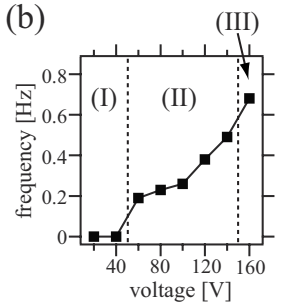

(e)

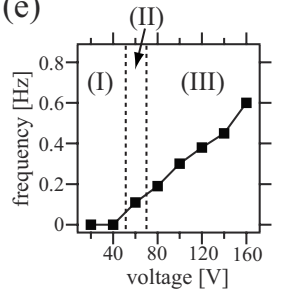

(c)

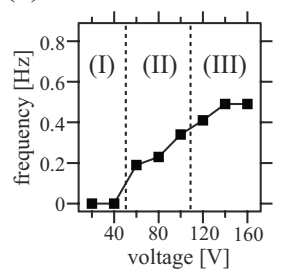

(f)

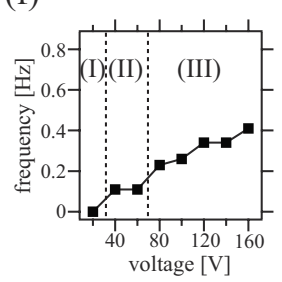

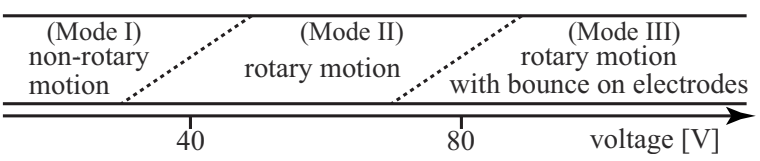

FIG. 3. [(a)-(f)] Dependence of the frequency of rotary motion on the applied voltage. (a) $r=14.0 \mu \mathrm{m}$. (b) $r=17.1 \mu \mathrm{m}$. (c) $r=18.8 \mu \mathrm{m}$. (d) $r=19.5 \mu \mathrm{m}$. (e) $r=31.8 \mu \mathrm{m}$. (f) $r=34.6 \mu \mathrm{m}$. Areas (I)-(III) correspond to the modes of motion in the phase diagram $(\mathrm{g})$. (g) Phase diagram of droplet motion depending on the applied voltage.

$\mathrm{V}$, droplets showed rotary motion with bouncing on the electrodes (Mode III). At approximately 40-80 V, droplets exhibited simple rotary motion (Mode II). However, at much lower voltages (approximately 20-40 V), droplets did not exhibit rotary motion (Mode I), which is plotted as $0 \mathrm{~Hz}$. The voltage-dependent behavior of a droplet is schematically illustrated as a phase diagram [Fig. 3(g)].

We propose a model to describe the rotary motion of w/o droplets. In an equation of motion at a micrometer scale, a viscosity term is more dominant than an inertia term because the Reynolds number, $R_{e}$, is very small; $R_{e}=\rho v r / \eta \sim 10^{-3}$ $\ll 1$, where $\rho\left(\sim 10^{3} \mathrm{~kg} / \mathrm{m}^{3}\right)$ and $\eta\left(\sim 10^{-3} \mathrm{~Pa} \mathrm{~s}\right)$ are the density and viscosity of the mineral oil, respectively, and $v\left(\sim 10^{-4} \mathrm{~m} / \mathrm{s}\right)$ and $r\left(\sim 10^{-5} \mathrm{~m}\right)$ are the velocity and diameter of the water droplet. Therefore, an over-damped equation of motion under a constant electric field, $\boldsymbol{E}$, is given by

$$
k \dot{\boldsymbol{x}}=q \boldsymbol{E}+\frac{1}{2} \alpha \nabla|\boldsymbol{E}|^{2},
$$

where $k\left(=6 \pi \eta r \sim 10^{-7} \mathrm{~kg} / \mathrm{s}\right)$ is a coefficient of viscosity resistance, and $k \dot{\boldsymbol{x}}$ represents the viscosity resistance for a moving droplet with diameter $r$ and velocity $\dot{\boldsymbol{x}} . q \boldsymbol{E}$ and $(1 / 2) \alpha \nabla|\boldsymbol{E}|^{2}$ indicate an electric force and a dielectric force acting on a droplet with charge $q$ and polarizability $\alpha\left(\sim 10^{-30} \mathrm{C} \mathrm{m}^{2} / \mathrm{V}\right)$.

To exhibit a limit cycle trajectory, the dissipation of energy is required. In the case of rotary motion of the droplet, the process of charging and discharging of the droplet is considered to play a role in the energy dissipation. For simplicity, we assume that the time-dependent rate of the charge, $q$, is described as follows:

$$
\dot{q}=\left\{\begin{array}{l}
+a \text { (on the positive electrode) }, \\
-a \text { (on the negative electrode) } \\
-b q \text { (in the other area), }
\end{array}\right.
$$

which means that the droplet is charged around the positive/ negative electrodes at a rate of $\pm a$, and discharges at a rate of $-b q$ in the absence of the electrodes. 
Figure 2(e) shows the results of a numerical simulation calculated using Eqs. (1) and (2) (see supplementary material ${ }^{17}$ about detailed parameters, conditions, etc.). The trajectory of the droplet in the simulation is a unidirectional rotary motion, which agrees with the experimental results. The frequency also corresponds to the experimental trend.

The dependence of the frequency of rotary motion on the applied voltage can be explained using this model. $|\boldsymbol{E}| \propto V$ and $f \sim|\dot{\boldsymbol{x}}| / L$, where $f$ is the frequency of rotary motion and $L$ is the circumference. Thus, from Eq. (1), the dependence of $f$ on $V$ can be derived as

$$
f \sim c_{1} V+c_{2} V^{2},
$$

where $c_{1}$ and $c_{2}$ are proportionality coefficients. Equation (3) means that the frequency increases with the applied voltage, as observed in Figs. 3(a)-3(f).

In summary, the droplet is first attracted to either electrode by dielectric force and is charged. After that, it is electrostatically repelled from the electrode and moves toward the opposite electrode due to electric force with discharging into the bulk oil phase. Again, the droplet is attracted to the opposite electrode by dielectric force. The switching between electric and dielectric forces caused by charge dissipation will generate unidirectional rotary motion because the electric and dielectric forces generally do not have the same direction. Thus, the placement of the two electrodes is important for generating the rotary motion; e.g., in a linear location, the droplet never exhibited a rotary motion. ${ }^{14} \mathrm{In}$ addition, as the model shows, any dielectric object can exhibit rotary motion; it will not be limited to a w/o droplet.

Basically, at a micrometer scale, it is difficult to generate unidirectional rotary motion because of the inhibition by environmental viscosity and frictional force at a rotary shaft of a motor. In addition, the inhibition remarkably increases with a decrease in the motor size. Therefore, the downsizing of a conventional rotary motor has limits. The rotary motion we observed may be important for the development of a contact- less rotary motor to extract work and energy at a micrometer scale. ${ }^{1,8}$ The present results are expected to stimulate the expansion of a micromechanical system.

We are grateful to Professor F. Arai (Tohoku University) for his helpful technical suggestions. We thank Dr. M. Ichikawa, Dr. S. M. Nomura, Dr. I. N. Motoike, Dr. M. Hishida (Kyoto University), Dr. Y. Sumino (The University of Tokyo), and Dr. K. Nagai (Ochanomizu University) for their helpful discussions. This work was supported by a Grant-in-Aid for Creative Scientific Research (Project No. 18GS0421) from the Japan Society for the Promotion of Science.

${ }^{1}$ E. M. Purcell, Am. J. Phys. 45, 3 (1977).

${ }^{2}$ D. G. Grier, Nature (London) 424, 810 (2003).

${ }^{3}$ J. Voldman, Annu. Rev. Biomed. Eng. 8, 425 (2006)

${ }^{4} \mathrm{P}$. Tabeling, Introduction to Microfluidics (Oxford University Press, New York, 2005).

${ }^{5}$ T. B. Jones, Electromechanics of Particles (Cambridge University Press, New York, 1995).

${ }^{6}$ D. R. Link, E. Grasland-Mongrain, A. Duri, F. Sarrazin, Z. Cheng, G. Cristobal, M. Marquez, and D. A. Weitz, Angew. Chem. Int. Ed. 45, 2556 (2006).

${ }^{7}$ S. Teh, R. Lin, L. Hung, and A. Lee, Lab Chip 8, 198 (2008).

${ }^{8}$ Y. Hiratsuka, M. Miyata, T. Tada, and T. Q. P. Uyeda, Proc. Natl. Acad. Sci. U.S.A. 103, 13618 (2006).

${ }^{9}$ B. Behkam and M. Sitti, Appl. Phys. Lett. 90, 023902 (2007).

${ }^{10}$ S. Hiyama, R. Gojo, T. Shima, S. Takeuchi, and K. Sutoh, Nano Lett. 9, 2407 (2009).

${ }^{11}$ M. G. L. van den Heuvel and C. Dekker, Science 317, 333 (2007).

${ }^{12}$ T. Mochizuki, Y. Mori, and N. Kaji, AIChE J. 36, 1039 (1990).

${ }^{13}$ J. Eow, M. Ghadiri, and A. Sharif, Colloids Surf., A 225, 193 (2003).

${ }^{14}$ M. Hase, S. N. Watanabe, and K. Yoshikawa, Phys. Rev. E 74, 046301 (2006).

${ }^{15}$ Y. Jung, H. Oh, and I. Kang, J. Colloid Interface Sci. 322, 617 (2008).

${ }^{16}$ W. D. Ristenpart, J. C. Bird, A. Belmonte, F. Dollar, and H. A. Stone, Nature (London) 461, 377 (2009).

${ }^{17}$ See supplementary material at http://dx.doi.org/10.1063/1.3358385 for a movie of the rotary motion of two droplets, and detailed description for the numerical simulation. 